1 Hacettepe Journal of Mathematics and Statistics

h Volume 46 (4) (2017), 613-620

\title{
Semi-strongly asymptotically non-expansive mappings and their applications on fixed point theory
}

\author{
Chris Lennard* and Veysel Nezir ${ }^{\dagger \ddagger}$
}

\begin{abstract}
We study fixed point theory for semi-strongly asymptotically nonexpansive and strongly asymptotically nonexpansive mappings. We consider these mappings for renormings of $l^{1}$ and $c_{0}$, and show that $l^{1}$ cannot be equivalently renormed to have the fixed point property for semi-strongly asymptotically nonexpansive mappings, while $c_{0}$ cannot be equivalently renormed to have the fixed point property for strongly asymptotically nonexpansive mappings. Next and more importantly, we show reflexivity is equivalent to the fixed point property for affine semi-strongly asymptotically nonexpansive mappings in Banach lattices. Finally, we give an application of our results in Lorentz-Marcinkiewicz spaces $l_{w, \infty}^{0}$, and some examples of these new types of mappings associated with a large class of $c_{0}$-summing basic sequences in $c_{0}$.
\end{abstract}

Keywords: nonexpansive mapping, reflexive Banach space, fixed point property, weak fixed point property, closed bounded convex set, asymptotically isometric $c_{0}$-summing basic sequence, Lorentz-Marcinkiewicz spaces, semi-strongly asymptotically nonexpansive mapping, strongly asymptotically nonexpansive mapping.

2000 AMS Classification: 47H09, 46B42, 46B45, 46B10.

Received : 28.08.2015 Accepted : 09.08.2016 Doi : 10.15672/HJMS.20174620775

*Department of Mathematics, The Dietrich School of Arts and Sciences, University of Pittsburgh, Pittsburgh, PA, 15260, USA., Email: lennard@pitt.edu

${ }^{\dagger}$ Department of Mathematics, Faculty of Science and Letters, Kafkas University, Kars, 36100, Turkey, Email: veyselnezir@yahoo.com

$\ddagger$ Corresponding Author. 


\section{Introduction}

Let $(X,\|\cdot\|)$ be a Banach space. Let $C$ be a non-empty subset of $X$. We recall that a mapping $T: C \rightarrow C$ is nonexpansive if $\|T x-T y\| \leq\|x-y\|$ for all $x, y \in C$. We say that a Banach space $X$ has the fixed point property if every nonexpansive mapping $T$ defined from any closed, bounded, convex subset $C$ of $X$ into $C$ has a fixed point. Banach spaces with this property are said to have the "fixed point property for nonexpansive mappings". We often abbreviate this to $\operatorname{FPP}($ n.e. $)$. Moreover, $(X,\|\cdot\|)$ is said to have the "weak fixed point property for nonexpansive mappings" (w-FPP(n.e.)) if for every weakly compact, convex (non-empty) subset $C$ of $(X,\|\cdot\|)$, for all nonexpansive mappings $T: C \rightarrow C, T$ has a fixed point in $C$. The sequence spaces $\left(c_{0},\|\cdot\|_{\infty}\right)$ and $\left(l^{1},\|\cdot\|_{1}\right)$ are both nonreflexive and possess the w-FPP(n.e.), but do not have the $\operatorname{FPP}($ n.e. $)$.

Alspach [1] provided the first example of a fixed point free nonexpansive mapping on a weakly compact subset of Banach space $(X,\|\cdot\|)=\left(L^{1}[0,1],\|\cdot\|_{1}\right)$. Maurey showed $\left(c_{0},\|\cdot\|_{\infty}\right)$, the Banach space of real-valued sequences that converge to zero (with the absolute supremum norm $\left.\|\cdot\|_{\infty}\right)$, as well as reflexive subspaces of $L^{1}[0,1]$, have the wFPP. Dowling and Lennard [4] showed every nonreflexive subspace of $L^{1}[0,1]$ fails the fixed point property, providing a converse to the second of Maurey's results mentioned above.

In 2008, P.K. Lin [11] proved that there exists a non-reflexive Banach space $(X,\|\cdot\|)$ that has the fixed point property for nonexpansive mappings. Lin's example is $\left(\ell^{1},\|\cdot\|_{1}\right)$ with an equivalent norm, $\||\cdot \||$, where

$$
\left\||x \||=\sup _{n \in \mathbb{N}} \gamma_{n} \sum_{k=n}^{\infty}\left|x_{k}\right|, \text { for all } x=\left(x_{n}\right)_{n \in \mathbb{N}} \in \ell^{1}\right.
$$

Here, $\left(\gamma_{n}\right)_{n \in \mathbb{N}}$ is some sequence in $(0,1)$ that is strictly increasing to 1 . Lin used $\gamma_{n}:=$ $8^{n} /\left(1+8^{n}\right)$, for all $n \in \mathbb{N}$.

In 2009, Domínguez Benavides [3] proved: [Given a reflexive Banach space $(X,\|\cdot\|)$, there exists an equivalent norm $\|\cdot\|_{\sim}$ on $X \operatorname{such}$ that $\left(X,\|\cdot\|_{\sim}\right)$ has the fixed point property for nonexpansive mappings]. In 2014, motivated by work in Nezir [15] (which we will describe in this paper), Lennard and Nezir [10] used the above-described theorem of Domínguez Benavides and the Strong James' Distortion Theorems [5, Theorem 8], to prove the following theorem: [If a Banach space is a Banach lattice, or has an unconditional basis, or is a symmetrically normed ideal of operators on an infinite-dimensional Hilbert space, then it is reflexive if and only if it has an equivalent norm that has the fixed point property for cascading nonexpansive mappings].

In this paper, we prove that $l^{1}$ cannot be equivalently renormed to have the FPP for semi-strongly asymptotically nonexpansive maps. Moreover, by a similar proof to that of Theorem 10 of Dowling, Lennard and Turett [5], we show that $c_{0}$ cannot be equivalently renormed to have the FPP for strongly asymptotically nonexpansive maps. From this, we conclude that if $(X,\|\cdot\|)$ is a non-reflexive Banach lattice, then $(X,\|\cdot\|)$ fails the fixed point property for $\|\cdot\|$-semi-strongly asymptotically nonexpansive mappings.

We strengthen this result to: [If a Banach space is a Banach lattice then it is reflexive if and only if it has the fixed point property for affine semi-strongly asymptotically nonexpansive mappings].

In fixed point theory, we are interested in Banach spaces $(X,\|\cdot\|)$ that contain subspaces isomorphic to $c_{0}$. Also, we are interested in Banach spaces that contain " $c_{0^{-}}$ summing basic sequences." (See Section 5 for the definition.) Reflexive Banach spaces $(X,\|\cdot\|)$ (for example, $L^{p}, 1<p<\infty$, and Hilbert spaces) do not contain $c_{0}$-summing 
basic sequences. On the other hand, many non-reflexive Banach spaces do. LorentzMarcinkiewicz spaces $l_{w, \infty}^{0}$, to which we will apply our results in this paper, are of this type. (See Section 5.)

Also in Section 5, for a large family of $c_{0}$-summing basic sequences $\left(\eta_{n}\right)_{n \in \mathbb{N}}$ in $c_{0}$, on the set $E=\overline{\mathrm{co}}\left(\left\{\eta_{n}: n \in \mathbb{N}\right\}\right)$, we present an example of a fixed point free affine semistrongly asymptotically nonexpansive mapping. It is the right shift $T$ on $E$. This is an interesting and natural example of a fixed point free affine semi-strongly asymptotically nonexpansive mapping that is generally not nonexpansive.

See, for example, [9] and [10] for more definitions and other information on fixed point theory related to this paper. We remark that most of this work forms part of the Ph.D. Dissertation of the second author [15], written under the supervision of the first author.

\section{Preliminaries}

Let $\mathbb{N}$ denote the set of all positive integers. In this paper our scalar field is the real numbers, $\mathbb{R}$. If $E$ is a subset of a Banach space $(X,\|\cdot\|)$, we will denote the convex hull of $E$ by $\operatorname{co}(E)$ and the closed convex hull of $E$ by $\overline{\operatorname{co}}(E)$. As usual, $\|x\|_{\infty}:=\sup _{n \in \mathbb{N}}\left|x_{n}\right|$ for all $x=\left(x_{n}\right)_{n \in \mathbb{N}} \in c_{0}$; and $\|x\|_{1}:=\sum_{n=1}^{\infty}\left|x_{n}\right|$ for all $x=\left(x_{n}\right)_{n \in \mathbb{N}} \in \ell^{1}$, the Banach space of all absolutely summable scalar sequences.

2.1. Definition. Let $E$ be a non-empty closed, bounded, convex subset of a Banach space $(X,\|\cdot\|)$. Let $T: E \longrightarrow E$ be a mapping.

(1) We say $T$ is affine if [for all $\lambda \in[0,1]$, for all $x, y \in E, T((1-\lambda) x+\lambda y)=$ $(1-\lambda) T(x)+\lambda T(y)]$.

(2) We say $T$ is nonexpansive if $[\|T(x)-T(y)\| \leq\|x-y\|$, for all $x, y \in E]$. Also, we say that $E$ has the fixed point property for nonexpansive mappings if for all nonexpansive mappings $T: E \longrightarrow E$, there exists $z \in E$ with $T(z)=z$.

(3) We say $T$ is contractive if [ $\|T(x)-T(y)\|<\|x-y\|$, for all $x, y \in E$ with $x \neq y]$.

(4) We say $T$ is asymptotically nonexpansive if $\left[\left\|T^{n} x-T^{n} y\right\| \leq k_{n}\|x-y\|\right.$, for all $x, y \in E$ and for all $n \in \mathbb{N}$ ]; where $\left(k_{n}\right)_{n \in \mathbb{N}}$ is some sequence of real numbers converging to 1 .

(5) We say $T$ is strongly asymptotically nonexpansive if $\exists\left\{\beta_{n, m}: n, m \in \mathbb{N}, n \geq m \geq\right.$ $0\} \subseteq[1, \infty)$ such that $\left[\forall x, y \in E\right.$ and $\left.\forall n \geq m,\left\|T^{n} x-T^{n} y\right\| \leq \beta_{n, m}\left\|T^{m} x-T^{m} y\right\|\right]$, $\left[\beta_{n, m} \rightarrow 1\right.$ as $\left.n \geq m \rightarrow \infty\right]$, and $\left[\beta_{n, m} \rightarrow 1\right.$ as $\left.n \rightarrow \infty, \forall m\right]$.

(6) We say $T$ is semi-strongly asymptotically nonexpansive if $\exists\left\{\lambda_{n, m}: n, m \in \mathbb{N}, n \geq\right.$ $m \geq 0\} \subseteq[1, \infty)$ such that $\left[\forall x, y \in E\right.$ and $\left.\forall n \geq m,\left\|T^{n} x-T^{n} y\right\| \leq \lambda_{n, m}\left\|T^{m} x-T^{m} y\right\|\right]$, and $\left[\lambda_{n, m} \rightarrow 1\right.$ as $\left.n \geq m \rightarrow \infty\right]$.

Note that we may always let $\beta_{m, m}=\lambda_{m, m}=1$ in Conditions (5) and (6) above. Also, Property (5) implies (4), and (5) implies (6). Generally, (6) does not imply (4) (and therefore (6) does not imply (5)). To show this we present an interesting example, which also sheds extra light on Theorems 3.2 and 3.3 below.

2.2. Example. Consider the Banach space $\left(\ell^{1},\|\cdot\|_{1}\right)$ with an equivalent norm of the type used by P.K. Lin (see above): $\left\||x \||:=\sup _{n \in \mathbb{N}} \gamma_{n} \sum_{k=n}^{\infty}\left|x_{k}\right|\right.$, for all $x=$ $\left(x_{n}\right)_{n \in \mathbb{N}} \in \ell^{1}$. Here, $\left(\gamma_{n}\right)_{n \in \mathbb{N}}$ is any fixed sequence in $(0,1)$ that is strictly increasing to 1. Let us further assume that the auxiliary sequence $\left(\gamma_{n+1} / \gamma_{n}\right)_{n \in \mathbb{N}}$ is strictly decreasing, and that $\gamma_{1}>1 / 2$. Lin's example of $\left[\gamma_{n}:=8^{n} /\left(1+8^{n}\right)\right.$, for all $\left.n \in \mathbb{N}\right]$ satisfies all of these properties. Define the closed, bounded, convex subset $C$ of $\ell^{1}$ by $C:=$ $\left\{x=\left(x_{n}\right)_{n \in \mathbb{N}}\right.$ : each $x_{n} \geq 0$ and $\left.\sum_{n=1}^{\infty} x_{n}=1\right\}$, and the affine mapping $[T: C \longrightarrow C$ by $T x:=\left(0, x_{1}, x_{2}, x_{3}, \ldots\right)$, for all $\left.x \in C\right]$. It is easy to calculate that for all integers $n \geq m \geq 0$, for all $x, y \in C$,

$$
\left\|\left|T^{n} x-T^{n} y\left\|\mid \leq \frac{\gamma_{n+1}}{\gamma_{m+1}}\right\| x-y\|\| .\right.\right.
$$


One can check, using $x:=e_{1}$ and $y:=e_{2}$, that each of the Lipschitz constants $\lambda_{n, m}:=$ $\gamma_{n+1} / \gamma_{m+1}$ is smallest possible. Clearly, $\lambda_{n, m} \longrightarrow 1$ as $n \geq m \longrightarrow \infty$. So, $T$ is semistrongly asymptotically nonexpansive. On the other hand, $k_{n}:=\lambda_{n, 0}=\gamma_{n+1} / \gamma_{1} \longrightarrow$ $1 / \gamma_{1}>1$, as $n \longrightarrow \infty$. Thus, $T$ is not asymptotically nonexpansive. (Indeed, $T$ is not even eventually asymptotically nonexpansive.)

Next, for our main theorems (Theorems 3.4 and 4.2), we need the notion of a Banach lattice. See, for example, [13] Definition 1.a.1.

Also, we recall the following proposition that can be found in, for example, [2].

2.3. Proposition. A Banach space $E$ is reflexive if and only if its closed unit ball $B_{E}$ is weakly compact.

\section{Equivalent renormings of $l^{1}$ and $c_{0}$, reflexivity and FPP's}

It is well-known (Theorems 1.c.12 in [12] and 1.c.5 in [13] ) that a Banach space with an unconditional basis or a Banach lattice is reflexive if and only if it contains no isomorphic copies of $c_{0}$ or $l^{1}$. Hence, if it can be shown that neither $c_{0}$ nor $l^{1}$ can be equivalently renormed to have a certain fixed point property, it would follow that this fixed point property in either a Banach lattice or in a Banach space with an unconditional basis would imply reflexivity.

By strengthening James' Distortion Theorem in [8], Dowling, Lennard and Turett gave the following theorem, called the Strong James' Distortion Theorem [5, Theorem 8].

3.1. Theorem. A Banach space $X$ contains an isomorphic copy of $l^{1}$ if and only if, for every (or, for some) null sequence $\left(\varepsilon_{n}\right)_{n \in \mathbb{N}}$ in $(0,1)$, there exists a sequence $\left(x_{n}\right)_{n \in \mathbb{N}}$ in $X$ such that

$$
\text { (†) } \quad\left(1-\varepsilon_{k}\right) \sum_{n=k}^{\infty}\left|t_{n}\right| \leq\left\|\sum_{n=k}^{\infty} t_{n} x_{n}\right\| \leq \sum_{n=k}^{\infty}\left|t_{n}\right|,
$$

for all $\left(t_{n}\right)_{n \in \mathbb{N}} \in l^{1}$ and for all $k \in \mathbb{N}$.

Also, a Banach space $X$ contains an isomorphic copy of $c_{0}$ if and only if, for every (or, for some) null sequence $\left(\varepsilon_{n}\right)_{n \in \mathbb{N}}$ in $(0,1)$, there exists a sequence $\left(x_{n}\right)_{n \in \mathbb{N}}$ in $X$ such that

$$
\left(1-\varepsilon_{k}\right) \sup _{n \geq k}\left|t_{n}\right| \leq\left\|\sum_{n=k}^{\infty} t_{n} x_{n}\right\| \leq\left(1+\varepsilon_{k}\right) \sup _{n \geq k}\left|t_{n}\right|,
$$

for all $\left(t_{n}\right)_{n \in \mathbb{N}} \in c_{0}$ and for all $k \in \mathbb{N}$.

We are ready to prove the following fact.

3.2. Theorem. If $X$ is a Banach space containing an isomorphic copy of $l^{1}$, then there exists a closed, bounded, convex subset $E$ of $X$ and an affine semi-strongly asymptotically nonexpansive $T: E \rightarrow E$ such that $T$ has no fixed point. Consequently, $l^{1}$ cannot be equivalently renormed to have the fixed point property for affine semi-strongly asymptotically nonexpansive mappings.

Proof. Let $X$ be a Banach space containing an isomorphic copy of $l^{1}$, and fix a null sequence $\left(\varepsilon_{n}\right)$ in $(0,1)$. Then by the Strong James' Distortion theorem, there exists a sequence $\left(x_{n}\right)$ in $\mathrm{X}$ such that the inequalities $(\dagger)$ above hold.

Next consider $E:=\overline{\mathrm{co}}\left(\left\{x_{n}: n \in \mathbb{N}\right\}\right)$. Note that $E=\left\{\sum_{n=1}^{\infty} t_{n} x_{n}: 0 \leq t_{n}, \forall n \in \mathbb{N}\right.$, and $\left.\sum_{n=1}^{\infty} t_{n}=1\right\}$. Consider the right shift $T: E \longrightarrow E$ given by $T\left(\sum_{j=1}^{\infty} t_{j} x_{j}\right)=$ $\sum_{j=1}^{\infty} t_{j} x_{j+1}$. 
Fix arbitrary $x, y \in E$, so that $x=\sum_{n=1}^{\infty} t_{n} x_{n}$ and $y=\sum_{n=1}^{\infty} s_{n} x_{n}$, with each $t_{n}, s_{n} \geq 0$ and $\sum_{n=1}^{\infty} t_{n}=\sum_{n=1}^{\infty} s_{n}=1$. Let $\alpha_{n}:=t_{n}-s_{n}$. Then, for any $n \in \mathbb{N}$,

$$
\left\|T^{n}(x)-T^{n}(y)\right\|=\left\|\sum_{j=1}^{\infty} \alpha_{j} x_{j+n}\right\| \leq \sum_{k=n+1}^{\infty}\left|\alpha_{k-n}\right|=\sum_{j=1}^{\infty}\left|\alpha_{j}\right| .
$$

Now, fix $m \in \mathbb{N}$. We have that

$$
\left\|T^{m}(x)-T^{m}(y)\right\|=\left\|\sum_{j=m+1}^{\infty} \alpha_{j-m} x_{j}\right\| \geq\left(1-\varepsilon_{m+1}\right) \sum_{k=m+1}^{\infty}\left|\alpha_{k-m}\right| .
$$

Thus, for all $n>m$,

$$
\left\|T^{n}(x)-T^{n}(y)\right\| \leq \sum_{j=1}^{\infty}\left|\alpha_{j}\right| \leq \frac{1}{\left(1-\varepsilon_{m+1}\right)}\left\|T^{m}(x)-T^{m}(y)\right\| .
$$

We define $\lambda_{n, m}:=\frac{1}{1-\varepsilon_{m+1}}, \forall n \geq m \geq 1$. Then $\lambda_{n, m} \longrightarrow 1$ as $n \geq m \longrightarrow \infty$, while $\left[\left\|T^{n}(x)-T^{n}(y)\right\| \leq \lambda_{n, m}\left\|T^{m}(x)-T^{m}(y)\right\|\right.$ for all $\left.x, y \in E\right]$. Thus, $T$ is affine and $\|\cdot\|$-semi-strongly asymptotically nonexpansive, and it is easy to check that $T$ has no fixed point in $E$.

3.3. Theorem. If $X$ is a Banach space containing an isomorphic copy of $c_{0}$, then there exists a closed, bounded, convex subset $E$ of $X$ and an affine strongly asymptotically nonexpansive $T: E \rightarrow E$ such that $T$ has no fixed point. Consequently, $c_{0}$ cannot be equivalently renormed to have the fixed point property for affine strongly asymptotically nonexpansive mappings.

Proof. Let $X$ be a Banach space containing an isomorphic copy of $c_{0}$. Dowling, Lennard and Turett proved in [5] that a certain right shift mapping is asymptotically nonexpansive and fixed point free. We will use a similar method to show that a right shift mapping is strongly asymptotically nonexpansive. By Theorem 8 in [5], there exist a null sequence $\left(\varepsilon_{n}\right)_{n \in \mathbb{N}}$ and a sequence $\left(x_{n}\right)$ in $X$ so that the following strengthening of the inequalities ( ) above hold.

$$
\sup _{n \geq k}\left|t_{n}\right| \leq\left\|\sum_{n=k}^{\infty} t_{n} x_{n}\right\| \leq\left(1+\varepsilon_{k}\right) \sup _{n \geq k}\left|t_{n}\right|,
$$

for all $\left(t_{n}\right)_{n \in \mathbb{N}} \in c_{0}$ and for all $k \in \mathbb{N}$. Let $E:=\overline{\mathrm{co}}\left(\left\{x_{n}: n \in \mathbb{N}\right\}\right)$. Again let $T\left(\sum_{j=1}^{\infty} t_{j} x_{j}\right)=\sum_{j=1}^{\infty} t_{j} x_{j+1}$.

Fix arbitrary $x, y \in E$, so that $x=\sum_{n=1}^{\infty} t_{n} x_{n}$ and $y=\sum_{n=1}^{\infty} s_{n} x_{n}$, with each $t_{n}, s_{n} \geq 0$ and $\sum_{n=1}^{\infty} t_{n}=\sum_{n=1}^{\infty} s_{n}=1$. Let $\alpha_{n}:=t_{n}-s_{n}$. Then, for any $n \in \mathbb{N}$,

$$
\left\|T^{n}(x)-T^{n}(y)\right\|=\left\|\sum_{k=n+1}^{\infty} \alpha_{k-n} x_{k}\right\| \leq\left(1+\varepsilon_{n+1}\right) \sup _{j \geq 1}\left|\alpha_{j}\right| .
$$

Fixing $m \in \mathbb{N}$, we get

$$
\left\|T^{m}(x)-T^{m}(y)\right\|=\left\|\sum_{j=m+1}^{\infty} \alpha_{j-m} x_{j}\right\| \geq \sup _{j \geq 1}\left|\alpha_{j}\right| .
$$

Therefore, for all $n>m$,

$$
\left\|T^{n}(x)-T^{n}(y)\right\| \leq\left(1+\varepsilon_{n+1}\right) \sup _{j \geq 1}\left|\alpha_{j}\right| \leq\left(1+\varepsilon_{n+1}\right)\left\|T^{m}(x)-T^{m}(y)\right\| .
$$

Define $\beta_{n, m}:=\left(1+\varepsilon_{n+1}\right), \forall n \geq m \geq 0$. Then $\left[\beta_{n, m} \longrightarrow 1\right.$ as $\left.n \longrightarrow \infty, \forall m \in \mathbb{N}\right]$, $\left[\beta_{n, m} \longrightarrow 1\right.$ as $\left.n>m \longrightarrow \infty\right]$, and $\left[\left\|T^{n}(x)-T^{n}(y)\right\| \leq \beta_{n, m}\left\|T^{m}(x)-T^{m}(y)\right\|\right.$, for all 
$x, y \in E]$. Thus, $T$ is affine and $\|\cdot\|$-strongly asymptotically nonexpansive, and $T$ has no fixed point in $E$.

We may now conclude the following.

3.4. Theorem. Let $(X,\|\cdot\|)$ be a nonreflexive Banach lattice. Then, $(X,\|\cdot\|)$ fails the fixed point property for affine $\|\cdot\|$-semi-strongly asymptotically nonexpansive mappings.

Proof. Since $(X,\|\cdot\|)$ is a nonreflexive Banach lattice, $X$ contains an isomorphic copy of $l^{1}$ or $c_{0}$ (by [13] Theorem 1.c.5). Hence, $(X,\|\cdot\|)$ fails the fixed point property for affine $\|\cdot\|$-semi-strongly asymptotically nonexpansive mappings.

\section{Reflexivity iff the FPP for affine semi-strongly asymptotically nonexpansive mappings.}

We will use the following interesting theorem of Mil'man and Mil'man [14].

4.1. Theorem. Let $(X,\|\cdot\|)$ be a Banach space and $C$ be a weakly compact convex set in $X$. Let $U: C \rightarrow C$ be an affine norm-to-norm continuous map. Then $U$ has a fixed point in $C$.

4.2. Theorem. Let $(X,\|\cdot\|)$ be a Banach lattice. Then the following are equivalent:

(1) $X$ is reflexive

(2) For every closed, bounded, convex set $C$ contained in $X$, for every affine semi-strongly asymptotically nonexpansive mapping $U: C \rightarrow C, U$ has a fixed point in $C$.

Proof. First we will prove that $[\operatorname{not}(1)$ implies not(2)]. Assume not(1). By Theorem 3.4, there exists a closed, bounded, convex subset $K$ in $X$ and there exists an affine semistrongly asymptotically nonexpansive mapping $T: K \rightarrow K$ such that $T$ is fixed point free; i.e., not(2) holds.

Now we prove (1) implies (2). Assume (1). Since $X$ is reflexive, its closed unit ball $B_{X}$ is weakly compact by the Proposition 2.3 . Thus, every closed, bounded, convex set $C$ contained in $X$ must also be weakly compact. Hence, Theorem 4.1 implies the result.

4.3. Remark. Similarly, the above result is true for every Banach lattice $X$ with an equivalent norm $\|\cdot\|$ on $X$ that is not necessarily a Banach lattice norm; as well as for every Banach space $(X,\|\cdot\|)$ with an unconditional Schauder basis.

\section{Examples and an application.}

In this section we give examples of semi-strongly asymptotically mappings and strongly asymptotically mappings. We will not include proofs here. They are available in the Ph.D. Dissertation of the second author [15], and may also appear elsewhere.

A sequence $\left(x_{n}\right)_{n \in \mathbb{N}}$ in a Banach space $(X,\|\cdot\|)$ is a $c_{0}$-summing basic sequence if there exist constants $0<A \leq B<\infty$ such that for all $t=\left(t_{k}\right)_{k \in \mathbb{N}} \in c_{00}$,

$$
A \sup _{n \in \mathbb{N}}\left|\sum_{k=n}^{\infty} t_{k}\right| \leq\left\|\sum_{n=1}^{\infty} t_{n} x_{n}\right\| \leq B \sup _{n \in \mathbb{N}}\left|\sum_{k=n}^{\infty} t_{k}\right| .
$$

Here, $c_{00}$ is the vector space of all finitely non-zero scalar sequences.

Moreover, whenever the leftmost inequality above holds, we say that $\left(x_{n}\right)_{n \in \mathbb{N}}$ satisfies a lower $c_{0}$-summing estimate.

In 2004 Dowling, Lennard and Turett [6] proved that every non-weakly compact, closed, bounded, convex subset $K$ of $\left(c_{0},\|\cdot\|_{\infty}\right)$ is such that there exists a $\|\cdot\|_{\infty^{-}}$ nonexpansive mapping $T$ on $K$ that is fixed point free. (This is a converse to the first theorem of Maurey described in the Introduction.) In general the mapping in [6] failing 
to have a fixed point is not affine. Concentrating on this subject, Lennard and Nezir investigated whether there exists a fixed point free affine nonexpansive mapping on any non-weakly compact closed, bounded, convex subset of $c_{0}$. In 2011 they showed in [9] that very large classes of subsets in $c_{0}$ fail the FPP for nonexpansive and affine mappings. In particular, they proved: [if a Banach space contains an asymptotically isometric (a.i.) $c_{0}$-summing basic sequence $\left(x_{i}\right)_{i \in \mathbb{N}}$, then the closed convex hull of the sequence fails the FPP for affine contractive mappings]. We recall now the definition of an asymptotically isometric $c_{0}$-summing basic sequence in a Banach space $(X,\|\cdot\|)$. (See, for example, [6] or [9].)

5.1. Definition. Let $\left(x_{n}\right)_{n \in \mathbb{N}}$ be a sequence in a Banach space $(X,\|\cdot\|)$. We define $\left(x_{n}\right)_{n \in \mathbb{N}}$ to be an asymptotically isometric (ai) $c_{0}$-summing basic sequence in $(X,\|\cdot\|)$ if there exists a null sequence $\left(\varepsilon_{n}\right)_{n \in \mathbb{N}}$ in $[0, \infty)$ such that for all sequences $\left(t_{n}\right)_{n \in \mathbb{N}} \in c_{00}$,

$$
\sup _{n \geq 1}\left(\frac{1}{1+\varepsilon_{n}}\right)\left|\sum_{j=n}^{\infty} t_{j}\right| \leq\left\|\left|\sum_{j=1}^{\infty} t_{j} x_{j} \| \leq \sup _{n \geq 1}\left(1+\varepsilon_{n}\right)\right| \sum_{j=n}^{\infty} t_{j} \mid .\right.
$$

5.2. Theorem. (See [15]) Let $\left(b_{n}\right)_{n \in \mathbb{N}}$ and $\left(\gamma_{n}\right)_{n \in \mathbb{N}}$ be two sequences of positive real numbers with $b_{n} \underset{n}{\longrightarrow} 1$ and $\gamma_{n} \underset{n}{\longrightarrow} 1$, such that $\sigma:=\sum_{n=2}^{\infty}\left|\gamma_{n}-\gamma_{n-1}\right|<\infty$. We define the sequence $\left(\eta_{n}\right)_{n \in \mathbb{N}}$ in $c_{0}$ by setting

$$
\eta_{n}:=\gamma_{n}\left(b_{1} e_{1}+b_{2} e_{2}+b_{3} e_{3}+b_{4} e_{4}+\ldots .+b_{n} e_{n}\right), \text { for all } n \in \mathbb{N} .
$$

Also suppose $\left(\eta_{n}\right)_{n \in \mathbb{N}}$ satisfies a lower co-summing estimate. Let $E:=\overline{\mathrm{co}}\left(\left\{\eta_{n}: n \in \mathbb{N}\right\}\right)$. Then $\left(\eta_{n}\right)_{n \in \mathbb{N}}$ is an asymptotically isometric $c_{0}$-summing basic sequence; and so there exists an affine contractive map $U: E \rightarrow E$ without a fixed point.

Generally, $U$ is not the right shift mapping $T: E \longrightarrow E$, since $T$ may fail to be nonexpansive. Of course $T$ is fixed point free and affine.

Moreover, $T$ is always $\|\cdot\|_{\infty}$-semi-strongly asymptotically nonexpansive. Further, if $b_{n} \downarrow_{n} 1$ and $\gamma_{n} \downarrow_{n} 1$, then $T$ is $\|\cdot\|_{\infty}$-strongly asymptotically nonexpansive.

Next, for all $x \in c_{0}$, we will define $x^{\star}:=\left(x_{n}^{\star}\right)_{n \in \mathbb{N}}$ to be the so-called decreasing rearrangement of $|x|:=\left(\left|x_{n}\right|\right)_{n \in \mathbb{N}}$. See, for example, [6] for more details about decreasing rearrangements.

5.3. Definition. Fix an arbitrary sequence $w=\left(w_{n}\right)_{n \in \mathbb{N}}$ such that $w_{n}>0, \forall n \in \mathbb{N}$, and $1=w_{1} \geq w_{2} \geq w_{3} \geq \cdots \geq w_{n} \geq w_{n+1} \geq \ldots$ Also assume $w_{n} \longrightarrow 0$ as $n \longrightarrow \infty$ and $\sum_{n=1}^{\infty} w_{n}=\infty$. E.g., $w_{n}=\frac{1}{n}, \forall n \in \mathbb{N}$. For all $x=\left(x_{n}\right)_{n \in \mathbb{N}} \in c_{0}$, let $\|x\|_{w, \infty}:=\sup _{n \in \mathbb{N}} \frac{\sum_{j=1}^{n} x_{j}^{\star}}{\sum_{j=1}^{n} w_{j}}$, and

$$
l_{w, \infty}^{0}:=\left\{x=\left(x_{n}\right)_{n \in \mathbb{N}} \in c_{0} \mid \limsup _{n \rightarrow \infty} \frac{\sum_{j=1}^{n} x_{j}^{\star}}{\sum_{j=1}^{n} w_{j}}=0\right\} .
$$

The Banach space $\left(l_{w, \infty}^{0},\|\cdot\|_{w, \infty}\right)$ is an analogue of $\left(c_{0},\|\cdot\|_{\infty}\right)$. In particular, $l_{w, \infty}^{0}$ is separable. For more on the Lorentz-Marcinkiewicz spaces, $l_{w, \infty}^{0}$, see (for example) Lindenstrauss and Tzafriri [12]. In Nezir [15] it is proven that $\left(l_{w, \infty}^{0},\|\cdot\|_{w, \infty}\right)$ has the weak fixed point property for nonexpansive mappings, but fails the fixed point property for nonexpansive mappings.

5.4. Theorem. (See [15]) Let $Y$ be any closed, non-reflexive vector subspace of $l_{w, \infty}^{0}$. Then $Y$ contains an isomorphic copy of $c_{0}$, and so (by Theorem 3.3 above) $\left(Y,\|\cdot\|_{w, \infty}\right)$ fails the fixed point property for strongly asymptotically nonexpansive maps. 


\section{Acknowledgements}

The second author is supported by a TUBITAK (The Scientific and Technological Research Council of Turkey) 2232 grant (no: 114C132); and he thanks the first author, Chris Lennard, for helpful suggestions and discussions about future projects.

\section{References}

[1] Alspach D. E., A fixed point free nonexpansive map, Proceedings of the American Mathematical Society $\mathbf{8 2}$, no. 3, 423-424, 1981.

[2] Beauzamy B., Introduction to Banach spaces and their Geometry, 11 (Elsevier Science Pub. Co., 1982).

[3] Domínguez Benavides T., A renorming of some nonseparable Banach spaces with the Fixed Point Property, J. Math. Anal. Appl. 350 (2), 525-530, 2009.

[4] Dowling P. N. and Lennard C. J., Every nonreflexive subspace of $L_{1}[0,1]$ fails the fixed point property, Proceedings of the American Mathematical Society 125 (2), 443-446, 1997.

[5] Dowling P. N., Lennard C. J., and Turett B., Some fixed point results in $l^{1}$ and $c_{0}$, Nonlinear Analysis 39, 929-936, 2000.

[6] Dowling P. N., Lennard C. J., and Turett B., Weak compactness is equivalent to the fixed point property in $c_{0}$, Proc. Amer. Math. Soc. 132 (6) 1659-1666, 2004.

[7] van Dulst D., Equivalent norms and the fixed point property for nonexpansive mappings, J. London Math. Soc. 25 (2), 139-144, 1982.

[8] James R. C., Uniformly non-square Banach spaces, Ann. of Math. 80, 542-550, 1964.

[9] Lennard C. J. and Nezir V., The closed, convex hull of every ai $c_{0}$-summing basic sequence fails the fpp for affine nonexpansive mappings, J. Math. Anal. Appl. 381, 678-688, 2011.

[10] Lennard C. J. and Nezir V., Reflexivity is equivalent to the perturbed fixed point property for cascading nonexpansive maps in Banach lattices, Nonlinear Analysis: Theory, Methods \& Applications 95, 414-420, 2014.

[11] Lin P. K., There is an equivalent norm on $\ell_{1}$ that has the fixed point property, Nonlinear Analysis 68, 2303-2308, 2008.

[12] Lindenstrauss J. and Tzafriri L., Classical Banach spaces I: Sequence Spaces, Ergebnisse der Mathematik und ihrer Grenzgebiete, 92 (Springer-Verlag, 1977).

[13] Lindenstrauss J. and Tzafriri L., Classical Banach spaces II: Function Spaces, Ergebnisse der Mathematik und ihrer Grenzgebiete, 97 (Springer-Verlag, 1979).

[14] Mil'man D. P. and Mil'man V. D., Some properties of non-reflexive Banach spaces, Mat. Sb. (N.S.) 65, 486-497, 1964 (in Russian).

[15] Nezir V., Fixed Point Properties for $c_{0}$-like Spaces, Ph.D. thesis, University of Pittsburgh, 2012. 